\title{
Real-time feedback for x-ray adaptive optics with an interferometric absolute distance sensor array
}

Ernesto Abruña, Vivek Badami, Lei Huang, Mourad Idir

Ernesto Abruña, Vivek G. Badami, Lei Huang, Mourad Idir, "Real-time feedback for X-ray adaptive optics with an interferometric absolute distance sensor array," Proc. SPIE 10761, Adaptive X-Ray Optics V, 107610H (18 September 2018); doi: 10.1117/12.2323698

Event: SPIE Optical Engineering + Applications, 2018, San Diego, California, United States 


\title{
Real-time feedback for $x$-ray adaptive optics with an interferometric absolute distance sensor array \\ Ernesto Abruña ${ }^{a^{*}}$, Vivek G. Badami ${ }^{\mathrm{a}}$, Lei Huang ${ }^{\mathrm{b}}$ and Mourad Idir $^{\mathrm{b}}$ \\ ${ }^{a}$ Zygo Corporation, Middlefield, CT, USA \\ ${ }^{b}$ Brookhaven National Laboratory - NSLS II, Upton, NY, USA
}

\begin{abstract}
The need for smaller focal spot sizes to meet the demands for higher resolution imaging is driving the adoption of adaptive optics in x-ray beamlines. Closed-loop control of the mirror shape, position, and orientation can greatly enhance the performance of these optics by allowing for rejection of perturbations. We demonstrate the performance of an array of interferometric absolute position sensors as a means of providing real-time feedback on shape changes of the reflecting surface of a bimorph mirror by comparison to a Fizeau interferometer.
\end{abstract}

Keywords: in-situ metrology, bimorph mirror, Kirkpatrick-Baez (KB) mirror, adaptive optics, synchrotron optics, x-ray optics, absolute position sensor, fiber position sensor

\section{INTRODUCTION}

Advanced light sources continue to improve their facilities to deliver powerful and unique imaging and measurement tools to meet the science needs of tomorrow. The next evolutionary steps, diffraction limited storage rings (DLSRs) and free electron lasers (XFELs), will enable the user to perform higher resolution imaging $(<20 \mathrm{~nm})^{1}$ at smaller time scales. Additionally, as the diversity in the user base continues to grow, facilities will need to provide flexible and easy to use instrumentation to service an increasingly diverse and multi-disciplinary user base that is not expert in the operation of beamlines make experiments run smoothly and efficiently.

Adaptive optics, specifically piezo bimorph mirrors, can offer a single hardware solution to address these new requirements. Mimura et al have demonstrated the value of adaptive optics combined with in situ mirror shape feedback for producing sub-10 $\mathrm{nm}$ focused line widths ${ }^{2}$. Adaptive optics have also already been used to demonstrate so called "zoom" functionality ${ }^{3}$, enabling continuously variable spot sizes ranging from one micron to tens of nanometers. This functionality can enable smaller focal spot sizes and 'push-button' spot size selection, making multi-scale imaging investigations ${ }^{4}$ possible.

$\mathrm{X}$-ray optical system performance is generally sensitive to changes in the ambient environment as well as to heating by the optical beam; piezo bimorph mirrors also suffer from actuator drift over time. Real-time feedback is therefore paramount for both maintaining the shape of the mirror (and consequentially the beam) and for changing it dynamically on command. Many feedback devices today are invasive, either measuring the focused beam directly, interrupting the experiment, or consuming flux to measure the beam constantly, impacting beamline performance. For these reasons, realtime in situ metrology of the mirror shape is advantageous for maintaining and dynamically controlling the beam shape.

We propose such an in situ metrology system that is designed to directly monitor the reflecting surface of the mirror and compare the performance of this system to a Fizeau interferometer.

\section{MEASUREMENT PRINCIPLES}

\subsection{Metrology System Architecture}

The architecture of this measurement uses an array of measurement points to sample the shape of a deformable mirror. This technique has been used for both optical fabrication metrology ${ }^{5}$ as well as for in situ shape control for deformable optics in astronomy applications ${ }^{6}$. This technique has also been demonstrated in to the curvature measurement of $\mathrm{x}$-ray

\footnotetext{
* Corresponding author: ernesto.abruna@ ametek.com
}

Adaptive X-Ray Optics V, edited by Daniele Spiga, Hidekazu Mimura, Proc. of SPIE Vol. 10761, 107610H (C)The Authors. Published under a Creative Commons Attribution CC-BY 3.0 License · doi: 10.1117/12.2211588 
deformable optics ${ }^{7}$. The sensor used in this experiment is a fiber-based absolute position interferometric sensor, specifically, the ZPSTM Sensor System produced by Zygo Corporation.

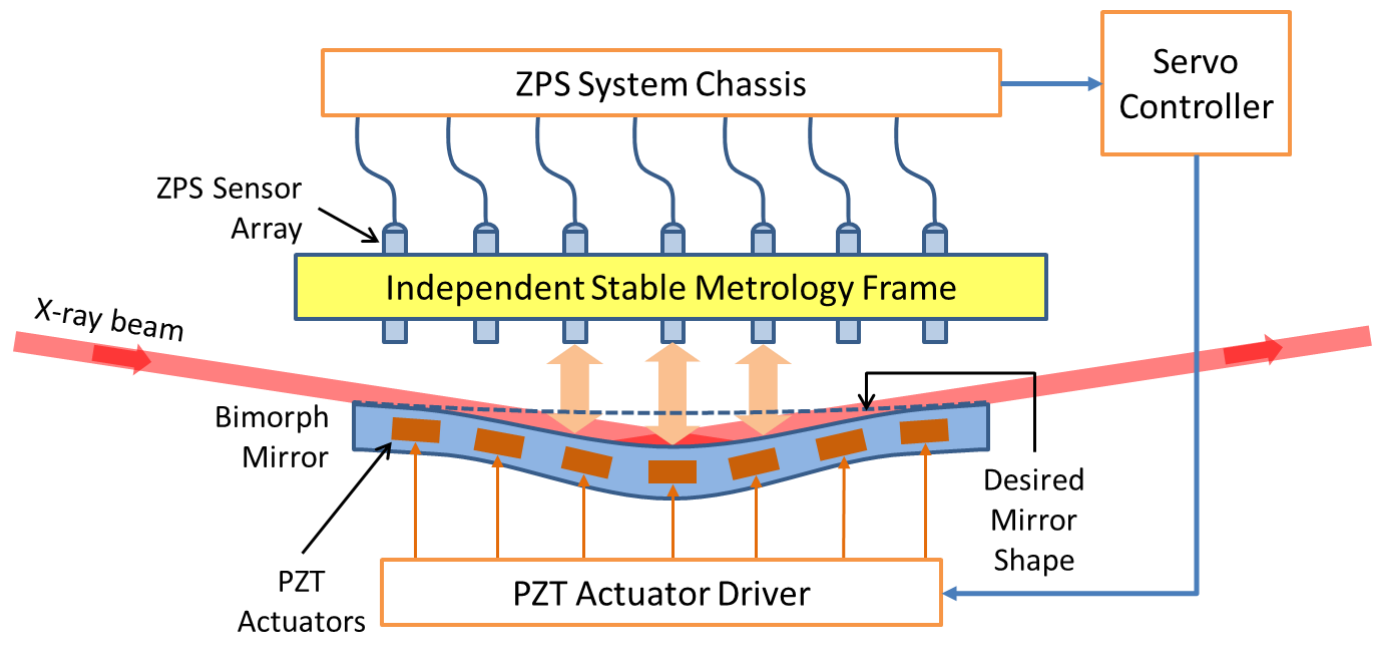

Figure 1. Architecture of a closed loop system for actively controlling the shape of a bimorph mirror

Figure 1 depicts the overall concept wherein an array of sensors mounted in an independent stable metrology frame measures the mirror surface at a series of points on the reflecting surface of the mirror. The metrology frame is held in a fixed relationship to the mirror surface at approximately the sensor working distance. In most hard x-ray optical systems, this spacing is sufficient to allow the x-ray beam to graze the mirror surface just below the face of the metrology frame, allowing the use of such a system for in situ monitoring during beamline operation. For x-ray optical systems operating at different wavelengths or with larger beams, a frame with a central aperture could be used with sensors positioned such that they straddle the beam.

Thermal and temporal stability of the metrology frame are key to the measurement, as is the mounting method of the metrology frame to ensures that mirror actuator motions do not produce deformations in the metrology frame. The signals from the array provide feedback for closed loop control of the mirror shape, enabling the maintenance and dynamic control of the beam size.
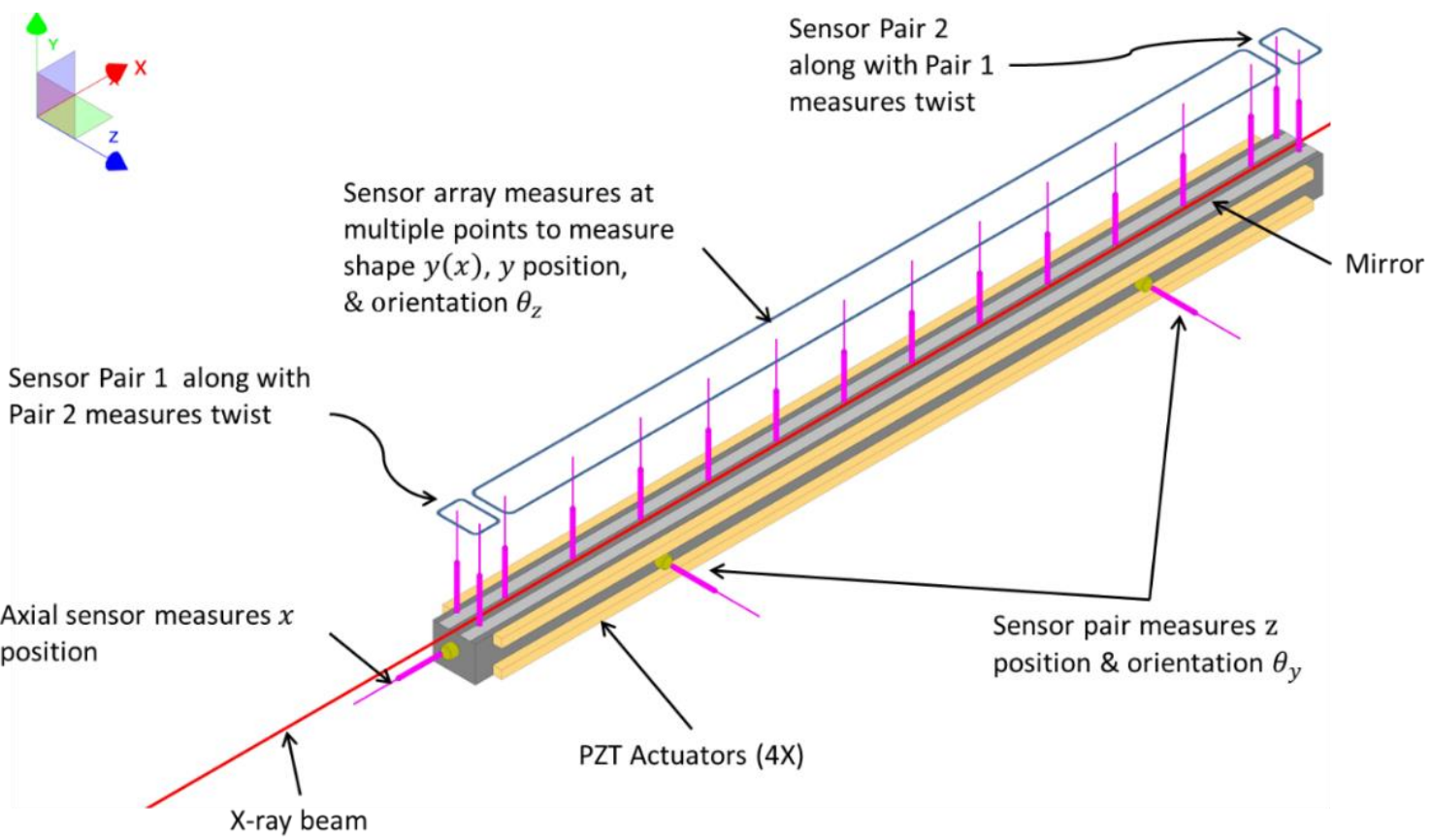

Figure 2. Measurement of shape and rigid-body motions. Mirror frame and metrology frame hidden for clarity. 
This concept can be extended to other non-rigid body motions (i.e. twist) as well as the rigid-body position and orientation of the mirror as shown in Figure 2 above. The absolute nature of the sensors enables the measurement of the absolute position and orientation of the mirror in up to all six degrees of freedom as well as the absolute shape and shape changes of the mirror. The sub-nm repeatability of the ZPS absolute position measurement makes possible the reestablishment of a previously optimized mirror shape and position after events such as a stripe or mirror swap, or an actuator power down.

\subsection{ZPS Sensor Technology}

The ZPS sensor functions in a Fizeau configuration as shown in Figure 3(a) where interference between the measurement reference surface and the target reflections generate the measurement signal. Most fiber position sensors can only measure target displacement $\Delta d$ and have no knowledge of the starting position; the ZPS sensor, in contrast, measures the actual starting distance $d$ from the sensor reference surface to the target, and any incremental changes $\Delta d$. This capacity, achieved through multi-wavelength techniques (illustrated in Figure 3(b)), provides a unique advantage in the metrology and control of bimorph mirrors.

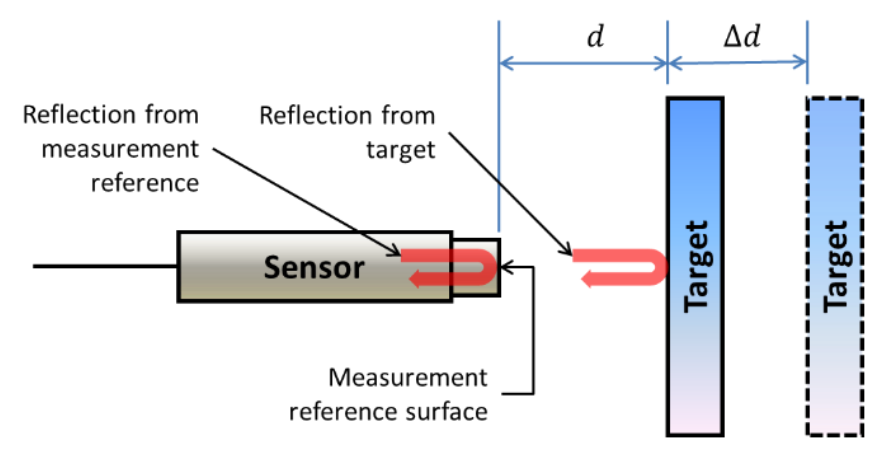

(a)

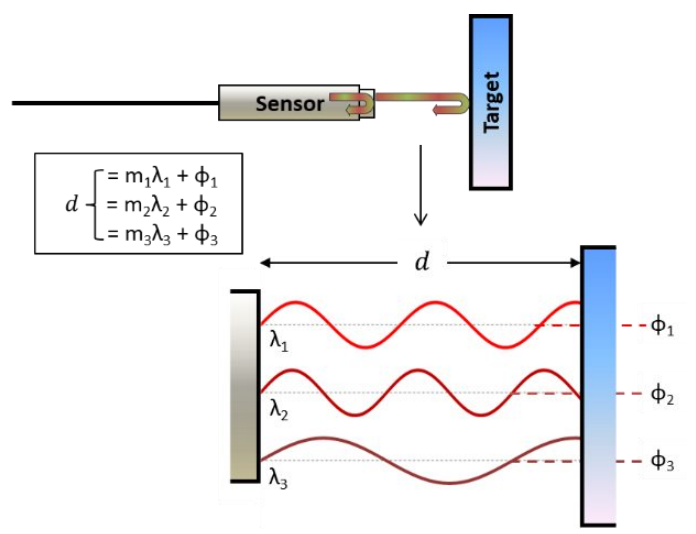

(b)

Figure 3. (a) Optical configuration of ZPS interferometric sensor, and (b) principle for absolute position determination through multi-wavelength measurement

The sensor itself, shown in Figure 4, is extremely compact which allows for the construction of an array of closely spaced sensors to enable sampling at a small spatial period. The noise performance of these sensors is $0.02 \mathrm{~nm} \mathrm{~Hz}{ }^{-1 / 2}(3 \sigma)$ over the full $3.5 \mathrm{~mm} \pm 0.6 \mathrm{~mm}$ measurement range. The ZPS sensor system was designed specifically for applications requiring tens of measurement channels (up to 64) ${ }^{8,9,10}$, making it well suited for measuring the shape, position, and orientation of multiple adaptive optical mirrors such as Kirkpatrick-Baez (KB) mirror pairs.

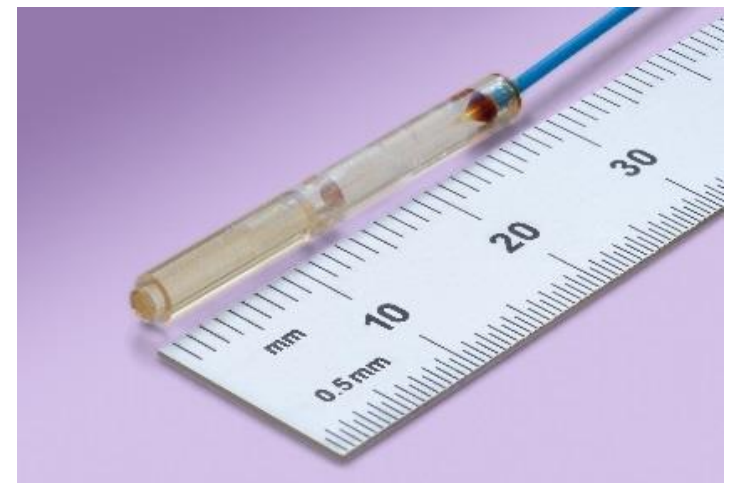

Figure 4. ZPS sensor image with length scale. The sensor measures $\varnothing 3 \mathrm{~mm}$ x $27 \mathrm{~mm}$. 


\section{EXPERIMENTAL SETUP}

An embodiment of the architecture outlined in section 2.1 was created for the experiment. The metrology frame is the key part of the setup and holds the two linear sensor arrays; each array is composed of 17 sensors spaced $\sim 23 \mathrm{~mm}$ apart. An aperture which provides access to the mirror surface for the simultaneous Fizeau measurement in compliance with the Abbe principle ${ }^{11}$ separates the two linear arrays. The metrology frame is constructed from $\mathrm{Al} 6061$ whose high thermal diffusivity minimizes frame deformation due to thermal gradients.

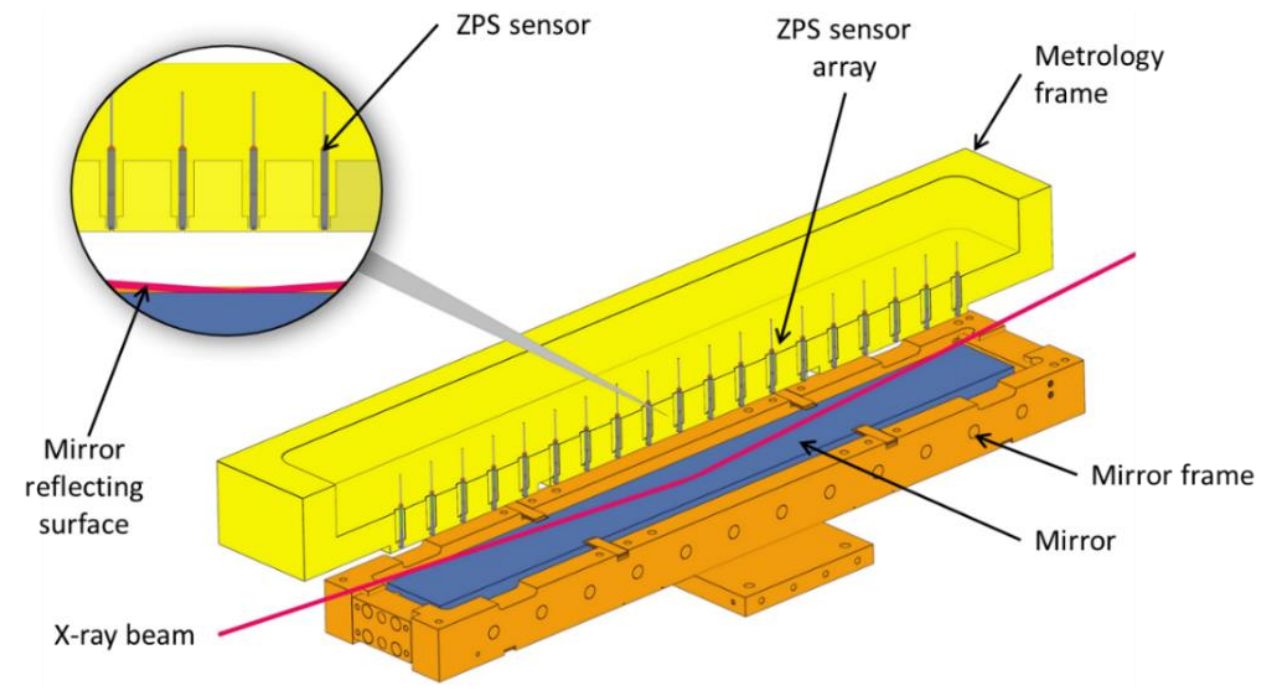

Figure 5. Metrology frame relative to the deformable mirrors. Frame is shown sectioned to reveal one of two linear arrays.

The full experimental setup is comprised of a common baseplate to which mount both the deformable mirror and the metrology frame. The metrology frame attaches kinematically to the baseplate through a set of three bipods, the design of which maximizes stability of the metrology frame shape by reducing deformations due to differential thermal expansion between the baseplate and the metrology frame. The entire baseplate is mounted vertically and positioned in front of an 18 " Fizeau interferometer. A simplified representation of this setup can be seen in Figure 6 below.

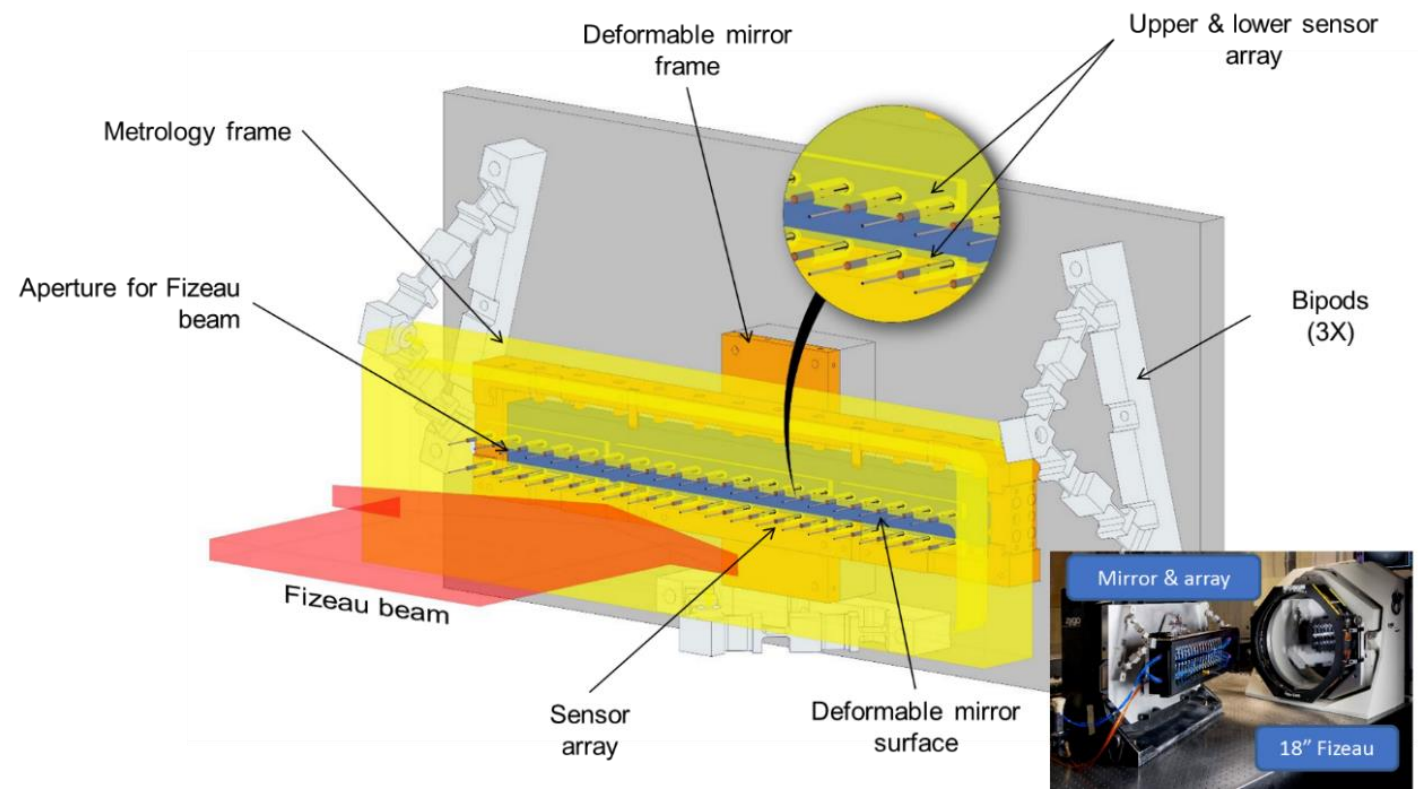

Figure 6. Simplified representation of the full measurement setup. Inset photograph shows the setup in front of an 18" Fizeau interferometer. Setup is rotated in the photograph to reveal details of the metrology frame. 
The mirror is a first generation bimorph which is comprised of piezo actuators sandwiched by two Zerodur® face sheets with 12 actuators distributed evenly along the length of the mirror $(\sim 450 \mathrm{~mm})$. The mirror mounts to an aluminum frame and bolts to a three degree-of-freedom adjustment which sets the relative angle between the mirror and the metrology frame as well as the spacing between them. This adjustment (not shown) is a classic three ball/three vee mount designed for the nanometer level stability required for this measurement.

\section{DATA ACQUISITION METHODS}

\subsection{Comparison Methodology}

The ZPS sensor array and Fizeau interferometer are compared by simultaneously measuring changes in the shape of the bimorph mirror. In each case, the Fizeau and sensor array measure the initial and final shapes of the mirror and the shape change is the difference between the initial and final shapes. The initial shape of the mirror in each case is its natural shape, i.e., shape with all actuators set to $0 \mathrm{~V}$.

The difference $\Delta S$ between the two measurements of shape change quantifies the agreement between the two methods. The differences between the two measurements were quantified at a variety of mirror deformations.

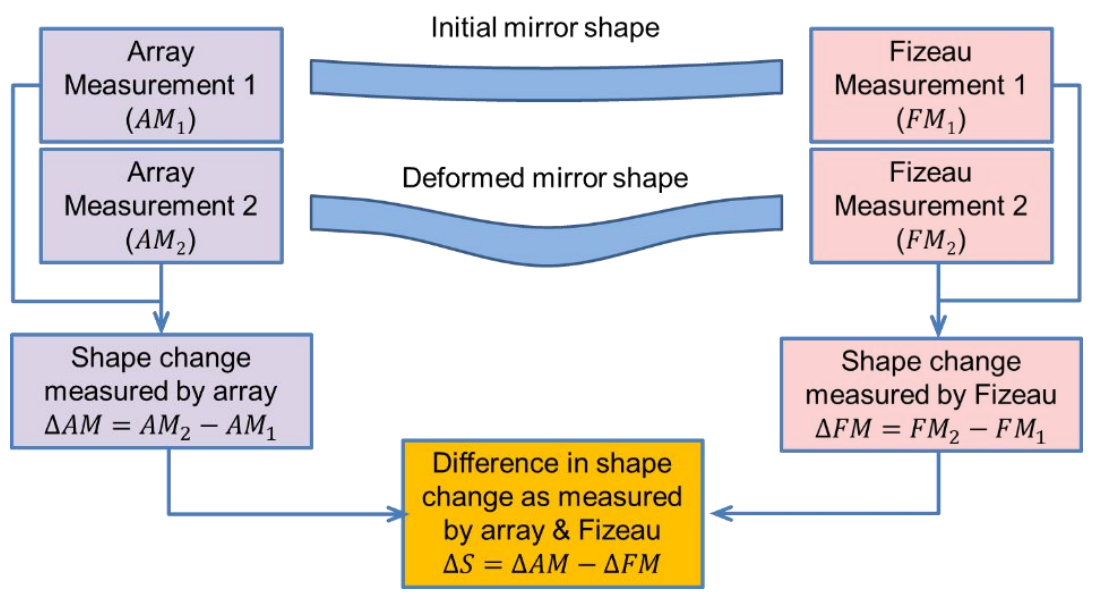

Figure 7. Illustration of data comparison.

\subsection{Data Acquisition \& Processing}

ZPS sensor data is processed through a $24 \mathrm{~Hz}$ low pass filter to minimize the effect of ambient noise while providing adequate bandwidth for the quasi-static mirror deformations. A dedicated refractometer that is part of the sensor array system corrects the array measurement for the effects of slow changes to temperature and pressure on the refractive index of air. Each sensor data point is an average of 10k points at a $2 \mathrm{kHz}$ sampling rate. A pairwise average of the two arrays produces an effective measurement at the same location as the center line of the Fizeau measurement.

The Fizeau data at each shape is the average of 32 phase measurements to minimize air turbulence effects. The data acquisition times of both the ZPS sensor and Fizeau interferometer are approximately equal $(\sim 5 \mathrm{sec})$. Each Fizeau data set is reduced to a line of data by taking the average value of 5 pixels around the centerline. The line of Fizeau data is aligned with the pairwise ZPS data and then subtracted to arrive at $\Delta S$.

\section{RESULTS}

Figures 8-10 show the mirror deflection and the difference data for a range of shapes and deflections. The graphic above the left-hand graph in each picture is a pictorial representation of the actuators that were energized during the test. Table 1 shows the RMS differences for the various cases. The short-term repeatability of the difference over the period of a typical measurement on a pointwise basis is $<0.4 \mathrm{~nm}$ 


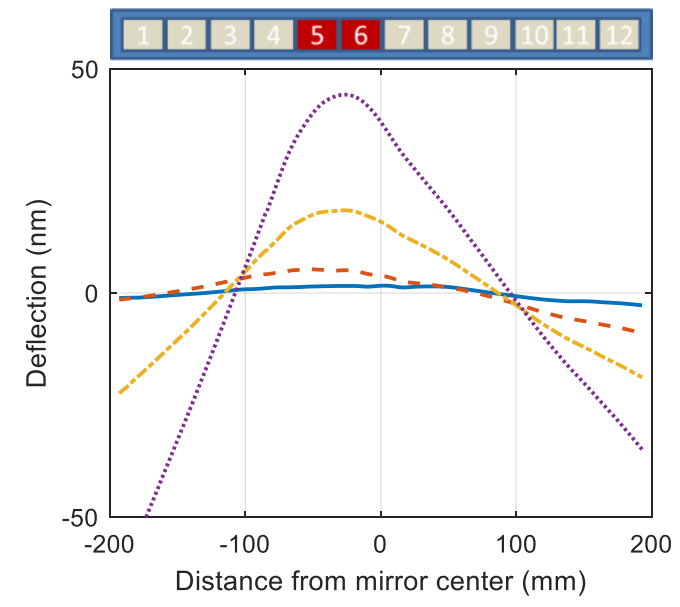

(a)

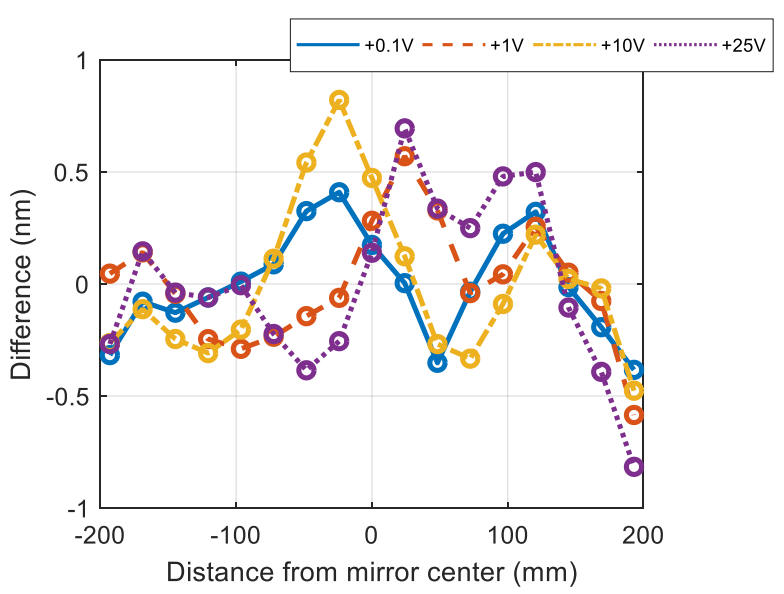

(b)

Figure 8. (a) Deflection $(\Delta \mathrm{FM})$ and (b) difference $(\Delta \mathrm{S})$ with actuators $5 \& 6$ activated

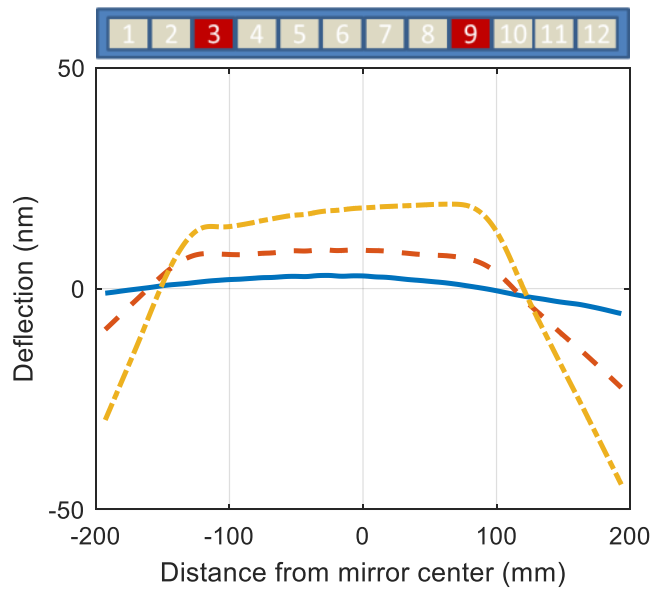

(a)

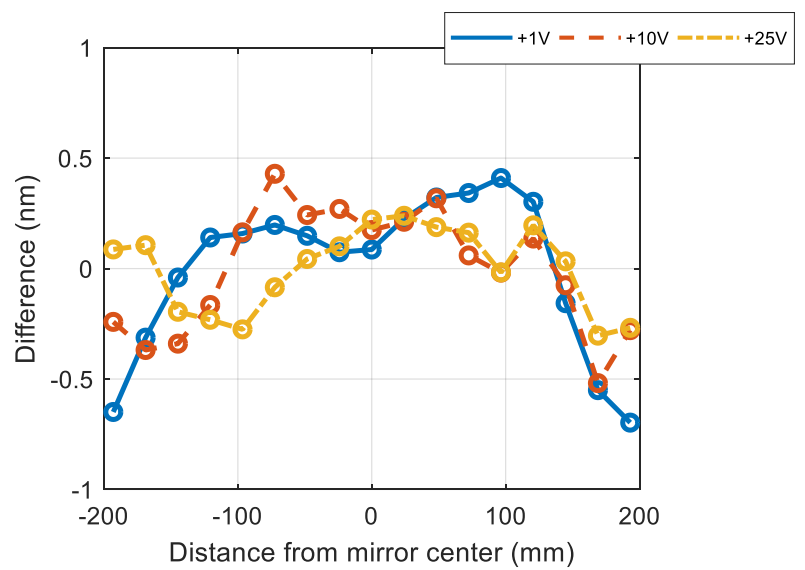

(b)

Figure 9. (a) Deflection $(\Delta \mathrm{FM})$ and (b) difference $(\Delta \mathrm{S})$ with actuators $3 \& 9$ activated

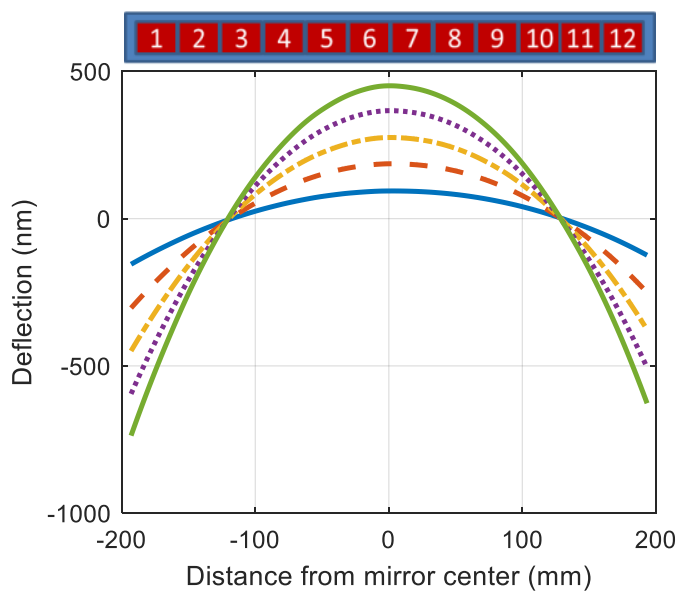

(a)

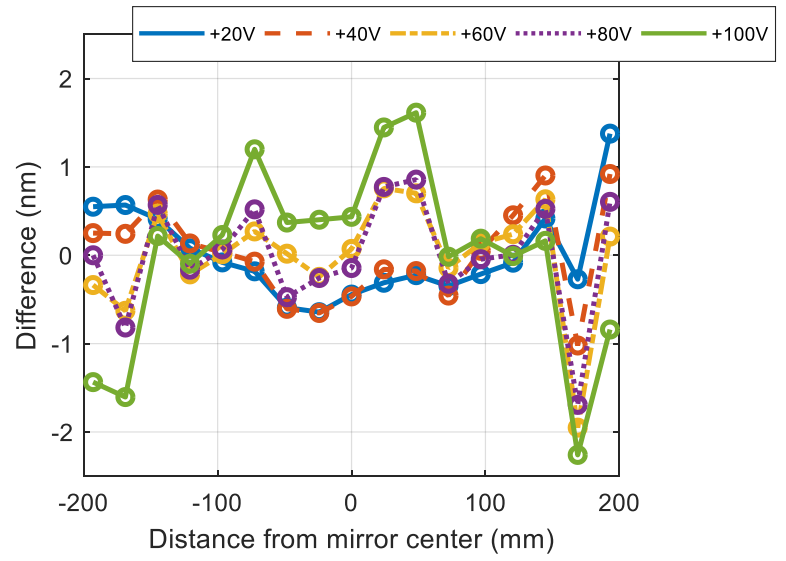

(b)

Figure 10. (a) Deflection $(\Delta \mathrm{FM})$ and (b) difference $(\Delta \mathrm{S})$ with all actuators activated 
Table 1. Summary of difference between Fizeau and sensor array mirror deflection measurements

\begin{tabular}{ccc}
\hline Piezos Actuated & Applied Voltage $(\mathbf{V})$ & Difference $(\mathbf{n m}$ RMS $)$ \\
\hline $5 \& 6$ & +0.1 & 0.23 \\
& +1 & 0.26 \\
& +10 & 0.34 \\
& +25 & 0.37 \\
& +1 & 0.34 \\
& +10 & 0.27 \\
All & +25 & 0.18 \\
& +20 & 0.52 \\
& +40 & 0.55 \\
& +60 & 0.59 \\
& +80 & 0.61 \\
\end{tabular}

\section{SUMMARY \& CONCLUSION}

The feasibility of sub-nm in situ mirror shape metrology has been demonstrated through a series of comparisons between the measurements of shape changes of a bimorph mirror by an array of ZPS ${ }^{\mathrm{TM}}$ sensors and a Fizeau interferometer. The comparisons show sub-nm agreement between the two measurement systems over a range of mirror shapes. The demonstrated performance of this proof-of-principle setup shows that it has the fidelity required to enable closed loop control of the mirror figure.

The repeatability of the measurements is dominated by air-turbulence effects in the Fizeau data due to the length of the cavity $(\sim 300 \mathrm{~mm})$. The results show low-order terms at the $\sim 2-3 \mathrm{~nm}$ level which may be due to deformations in the metrology frame as well as Fizeau errors resulting from the high fringe densities at higher-slope deflections. The overall measurement uncertainty for the point-wise difference varies with sensor position and mirror deflection and is estimated to be $1.5 \mathrm{~nm}(\mathrm{k}=2)$.

Improved results are expected by fabricating of the metrology frame using low thermal expansion material and/or operating in a high-vacuum typical of a beamline environment. Further experiments over a larger range of curvatures to show the metrological validity of this technique are ongoing.

\section{REFERENCES}

1. Advanced Photon Source Upgrade Project: Preliminary Design Report, Document Number: APSU-2.01-RPT-002, pg. 6-117. (Sept 2017)

2. Mimura, H. et al, "Breaking the 10nm barrier in hard X-ray focusing," Nature Physics 10, pp. 122-125 (2010).

3. Kimura, T., Matsuyama, S., Yamauchi, K. and Nishino, Y., "Coherent X-ray zoom condenser lens for diffractive and scanning microscopy," Optics Express 21, 9267-9276 (2013).

4. Advanced Photon Source Upgrade Project: Preliminary Design Report, Document Number: APSU-2.01-RPT-002, pp. 6-6 - 6-20. (Sept 2017)

5. Boland, R. J., "Using linear variable differential transformers and ultrasonic transducers to measure flatness and parallelism for NIF optics," Proc. SPIE 3782 (11 Nov 1999).

6. Biasi, R., Gallieni, D., Salinari, P., Riccardi, A. and Mantegazza, P., "Contactless thin adaptive mirror technology: past, present, and future," Proc. SPIE 7736, 77362B (2010).

7. Vannoni, M., Martín, I. F., Music, V. and Sinn, H., "Calibration and optimization of an x-ray bendable mirror using displacement-measuring sensors," Optics Express 24, 17292-17302 (2016).

8. Deck, L. L., "High-performance multi-channel fiber-based absolute distance measuring interferometer system", Proc. SPIE 7405, 74050E (21 Aug 2009).

9. Badami, V. G. and Abruña, E., "Absolutely: Small Sensor, Big Performance," Mikroniek 1, pp. 5-9 (2018).

10. http://www.zygo.com/zps/

11. Abbe, E., "Messapparate fur Physiker," Zeitschrift für Instrumentenkunde 10, 446-448 (1890). 